Journal for ImmunoTherapy of Cancer

\section{Lactoferrin-containing immunocomplex mediates antitumor effects by resetting tumor-associated macrophages to M1 phenotype}

To cite: Dong H, Yang Y, Gao C, et al. Lactoferrin-containing immunocomplex mediates antitumor effects by resetting tumor-associated macrophages to M1 phenotype. Journal for ImmunoTherapy of Cancer 2020;8:e000339. doi:10.1136/ jitc-2019-000339

- Additional material is published online only. To view please visit the journal online (http://dx.doi.org/10.1136/jitc2019-000339).

Accepted 01 March 2020
Check for updates

(C) Author(s) (or their employer(s)) 2020. Re-use permitted under CC BY-NC. No commercial re-use. See rights and permissions. Published by BMJ.

Institute of Biology and Medical Sciences, School of Biology and Basic Medical Science, Soochow University, Suzhou, China

\section{Correspondence to}

Dr Hongliang Dong, Soochow University, SuZhou, China; hldong@suda.edu.cn

Professor Xiaoming Gao, Soochow University, Suzhou, China; xmgao@suda.edu.cn

\section{ABSTRACT}

Background Tumor-associated macrophages (TAMs) resemble M2-polarized cells with potent immunosuppressive activity and play a pivotal role in tumor growth and progression. Converting TAMs to proinflammatory M1-like phenotype is thus an attractive strategy for antitumor immunotherapy.

Methods A mouse $\lg \mathrm{G}_{1}$ (kappa) monoclonal Ab, M-860, specific to human lactoferrin (LTF) was generated by using the traditional hybridoma cell fusion technology. TAMs were generated by culturing human and mouse $\mathrm{CD}_{14}{ }^{+}$monocytes in tumor-conditioned media containing a cytokine cocktail containing recombinant interleukin-4 (IL-4), interleukin-10 (IL-10) and macrophage colony stimulating factor (M-CSF). TAMs after treatment with immunocomplex (IC) between human LTF and M860 (LTFIC) were phenotypically and functionally characterized by flow cytometry (FACS), ELISA, Q-PCR and killing assays. The antitumor effects of LTF-IC were further analyzed using in vivo experiments employing tumor-bearing human FcyRlla-transgenic mouse models.

Results Through coligation of membrane-bound CD14 and FCYRIlla, LTF-IC rendered TAMs not only M2 to M1 conversion, evidenced by increased tumor necrosis factor $\alpha$ production, down-regulated M2-specific markers (CD206, arginase-1 and vascular endothelial growth factor) and upregulated M1-specific markers (CD86 and HLA-DR) expression, but also potent tumoricidal activity in vitro. LTF-IC administration conferred antitumor protective efficacy and prolonged animal survival in Fc $\gamma R$ Rllatransgenic mice, accompanied by accumulation of M1-like macrophages as well as significantly reduced infiltration of immunosuppressive myeloid-derived suppressor cells and regulatory T cells in solid tumor tissues.

Conclusions LTF-IC is a promising cancer therapeutic agent capable of converting TAMs into tumoricidal M1-like cells.

\section{INTRODUCTION}

Macrophages exist in at least two distinct states of polarized activation: the classically activated M1 and the alternatively activated M2 status. ${ }^{1-5}$ Bacterial lipopolysaccharide (LPS) and cytokines like IFN- $\gamma$, GM-CSF and tumour necrosis factor $\alpha$ (TNF- $\alpha)$ polarize macrophages toward the M1 phenotype. In contrast, IL-4, IL-13, IL-10 and IL-6 drive the polarization of macrophages towards M2 phenotype. $^{2}{ }^{3} 5$ Phenotypically, M2 macrophages show: (1) more phagocytic activity; (2) higher expression of scavenging (CD163), mannose (CD206) and galactose receptors; (3) production of ornithine and polyamines through the arginase pathway; (4) high IL-10 expression and low IL-12 expression. ${ }^{125} \mathrm{M} 1$ and M2 macrophages also exhibit distinct features in terms of metabolism of iron, folate and glucose. ${ }^{1}$ Functionally, M2 macrophages are considered immunoregulatory, while M1 macrophages proinflammatory. Unlike 'permanently differentiated' T helper-type 1 (Th1) or Th2 cells, macrophages exhibit high plasticity in M1-M2 transition depending on the microenvironment. ${ }^{23}$ Given that M1 and M2 cells exhibit antitumor and protumor potentials, respectively, targeted polarization of macrophages to M1 phenotype would provide potential therapeutic value for cancers. ${ }^{6}$

Macrophages are particularly abundant among infiltrating immune cells in solid tumor tissues. ${ }^{6-8}$ Blood monocytes infiltrate tumor tissues under the influence of chemokine CCL2, and are then induced to differentiate into mature macrophages by tumor microenvironmental signals including IL-3 and CSF-1, and eventually polarized by IL-4 and/or IL-10 as tumor-associated macrophages (TAMs). ${ }^{8-10}$ TAMs usually exhibit a M2 phenotype with potent immunosuppressive activity and play a pivotal role in tumor progression, metastasis and recurrence after treatment. ${ }^{8} 1112$ Multiple mechanisms have been proposed for TAMs to suppress cytotoxicity of tumor-infiltrating $\mathrm{T}$ and NK cells, including cell-cell contact through programmed cell death protein 1 (PD-1), 
cytotoxic T lymphocyte associated antigen 4 (CTLA-4) receptors, ${ }^{813} 14$ FAS ligand (FASL), TNF related apoptosis inducing ligand (TRAIL) and non-classical HLA-G and HLA-E ${ }^{15}$ and releasing regulatory signals such as IL-10, TGF- $\beta$, CCL5 $/ 20 / 22^{16}$ and arginase-1 (ARG- 1 ). ${ }^{17}{ }^{18}$ Additionally, TAMs also secrete vascular endothelial growth factor (VEGF) to directly support tumor growth by inducing neovascularization. ${ }^{1719}$

Much effort has been made on converting M2-polarized immunosuppressive TAMs to proinflammatory M1-like phenotype for the treatment of cancers in the recent years. ${ }^{80-25}$ Pharmacological blockade of CXCR2 receptor by a selective antagonist promoted the reeducation of TAMs toward a proinflammatory phenotype, leading to senescence and tumor inhibition in prostate cancer. ${ }^{22}$ Intravenous $\mathrm{IgG}$ impaired tumor progression and metastasis in a Fc receptor-dependent and macrophagedependent manner, attributable to intravenous IgG skewing of M2 macrophages toward M1 cells. ${ }^{26}$ Additionally, particulate yeast-derived $\beta$-glucan converted TAMs into immune-stimulating phenotype through the Dectin1-Syk-Card9-Erk pathway, leading to reduced tumor progression. ${ }^{27}$ Combination of CD40 agonism and CSF-1 receptor blockade modulated endogenous antitumor immunity and controlled tumor growth by promoting a proinflammatory phenotype in TAMs. ${ }^{28}$ Moreover, antimalarial drug chloroquine could also function as an antitumor agent, which is likely related to its ability to switch TAMs from M2 to tumor-killing M1 phenotype. ${ }^{29}$

Lactoferrin (LTF), first identified as an iron-binding protein, exerts diverse biological functions including antitumor effects. ${ }^{30-34}$ M860 is a mouse antihuman LTF monoclonal antibody $(\mathrm{mAb})$, prepared in this laboratory, which recognizes a conformation epitope and is able to form stable immunocomplex (IC) with LTF. ${ }^{32}{ }^{35}$ We have also shown that LTF-containing IC (LTF-IC) is able to switch human macrophages from M2 to M1 phenotype. ${ }^{36}$ We, therefore, wondered if LTF-IC is able to exert a similar effect on TAMs and suppress tumor growth in vivo. In the present study, we demonstrate that LTF-IC promoted M2-to-M1 switch of TAMs in vitro and significantly inhibited tumor progression and lengthened overall survival of tumor-bearing mice in vivo. These results suggest potential clinical application of LTF-IC in antitumor therapies.

\section{MATERIALS AND METHODS \\ Macrophage differentiation and analysis}

Peripheral blood mononuclear cells (PBMCs) were isolated from heparinized peripheral blood from healthy donors by density gradient centrifugation. All donors gave written informed consent to participate in the study. Monocytes were purified from PBMC by magnetic cell sorting using CD14 microbeads (Miltenyi Biotec, Germany), and used for generation of M1, M2 macrophages. Monocytes were cultured for 6 days in RPMI 1640 (Hyclone) containing 10\% fetal bovine serum (FBS, Biological Industries) supplemented with $20 \mathrm{ng} / \mathrm{mL}$ recombinant human M-CSF (Peprotech). At day 3, half of the medium was replaced by new medium containing cytokines. At day 7 , the medium was totally replaced in the presence of $20 \mathrm{ng} / \mathrm{mL}$ recombinant human IFN- $\gamma$ (Peprotech) for M1, and IL-4 (Peprotech) for M2, respectively.

TAMs derived human blood monocytes or mouse bone marrow cells were in vitro exposed to cancer cell (MDA-MB-231 or B16)-conditioned media plus the cytokine cocktail of IL-4, IL-10, and M-CSF according to the protocol of Benner et al. ${ }^{37}$ Differentiated TAMs, named MDA-MB-231-TAMs or B16-TAMs, were analyzed for phenotype (CD14, CD206, CD86), cytokine production (TNF- $\alpha$, IL-10), and gene expression (CD206, VEGF, ARG-1).

\section{The preparation of LTF-IC}

Preparation and characterization of a mouse mAbs against huLTF (M860) in this laboratory have been reported in our previous work. ${ }^{35}$ For preparation of LTF-IC, huLTF $(2 \mu \mathrm{g} / \mathrm{mL}$, Sigma-Aldrich) and M860 $(2 \mu \mathrm{g} / \mathrm{mL}, \mathrm{mAbs}$ of LTF), purified with protein $\mathrm{G}$ antibody affinity chromatography (GE Healthcare Life Sciences), or LTF-Abs were mixed in a sterile tube with gentle rotation at $37^{\circ} \mathrm{C}$ for an hour. ICs between LTF and M860 were separated from the uncoupled $\mathrm{Ab}$ and antigen using Sephadex Superfine G-75 column. The elutions of IC were pooled, desalted and concentrated. Endotoxin was removed by polymyxin $\mathrm{B}$ coupled beads repeatly and the level of endotoxin in IC was below $1 \mathrm{EU} / \mathrm{mg}$ which was detected by Chromogenic LAL Endotoxin Assay Kit (Genscript). ICs between ovalbumin (OVA) (Sigma-Aldrich) and M562 (mAbs of OVA) were used as control and prepared similarly.

\section{Mouse models of B16 melanoma}

Wild type (WT) and human CD32a (hCD32a)-transgenic mice (hCD32a-TG, from Jackson Laboratory), 6-8 weeks, were injected s.c. with $10^{6} \mathrm{~B} 16$ cells in $0.1 \mathrm{~mL}$ phosphatebuffered saline (PBS) on day 0. LTF, M860, LTF-IC (10 mg $\mathrm{kg}^{-1}$ ) or PBS were injected i.p. on day $-1,7$ and 14. Mice were inspected daily, and the tumor volume was measured as width $^{2} \times$ length $/ 2$. The survival of tumor-bearing mice was observed every day as well. The phenotype of tumor-infiltrating TAMs $\left(\mathrm{CD} 11 \mathrm{~b}^{+} \mathrm{CD} 11 \mathrm{c} \mathrm{F} 4 / 80^{+} \mathrm{Gr}-1^{-}\right)$was analyzed by FACS and Q-PCR. C57BL/6 mice bearing subcutaneous B16 melanoma received intratumor injection with either PBS, LTF-IC-treated B16-TAMs, B16-TAMs or M1 (IFN- $\gamma$ polarized) cells. On day 18 , the mice were killed to analyze the percentage of MDSCs and Tregs.

\section{Stimulation}

MDA-MB-231-TAMs and B16-TAMs were harvested by gentle pipetting and stimulated $\left(3 \sim 5 \times 10^{4}\right.$ cells/well $)$ with $30 \mu \mathrm{g} / \mathrm{mL}$ LTF, M860, LTF-IC and $100 \mathrm{ng} / \mathrm{mL}$ LPS (from Escherichia coli 0111:B4; Sigma-Aldrich) in 96-well plates (Nunc) for 24 hours in a $5 \% \mathrm{CO}_{2}$ incubator at $37^{\circ} \mathrm{C}$, then the supernatants were collected and stored at $4^{\circ} \mathrm{C}$, until analysis by ELISA. Cells were harvested and the changes 
of phenotype were determined by FACS analysis and Q-PCR.

\section{Antibodies and inhibitors blockade}

Blocking antibodies were preincubated with TAMs for 2 hours in a $5 \% \mathrm{CO}_{2}$ incubator at $37^{\circ} \mathrm{C}$, after which stimuli and culture medium were added resulting in a final antibody concentration of $5 \mu \mathrm{g} / \mathrm{mL}$ anti-CD16/32/64 and $2 \mu \mathrm{g} / \mathrm{mL}$ anti-CD14. Granzyme B (GzmB) was inhibited by incubating TAMs with titration of Z-AAD-CMK (invitrogen) for 2 hours before LTF-IC treatment.

\section{ELISA and NO assay}

The levels of TNFo, IL-10 in cell culture supernatants were measured by ELISA kits (eBioscience) according to the manufacturer's instructions. Standard curves were established using human recombinant cytokines provided in the kits. For NO-level assay, total nitrite/nitrate was measured in cell supernatants by using Nitric Oxide Colorimetric Assay Kit (BioVision, K262) according to the suppler's instructions.

\section{Flow cytometry}

For surface marker analysis, cells were collected, washed with PBS, and the pellets were incubated for $30 \mathrm{~min}$ at $4^{\circ} \mathrm{C}$ with anti-hCD14-APC, CD16-PE, CD32-PE, CD64-PE, CD86-PE, CD163-APC-Cy7, CD206-FITC, HLA-DR-PE or anti-mouse F4/80-APC-Cy7, CD11b-AF700, CD206-FITC, and CD86-PE, or APC, PE, FITC, APC-Cy7-conjugated isotype control Abs. The concentration at each antibody was used as the product protocol recommended. All surface flow cytometry antibodies were purchased from Biolegend, and in some cases anti-mouse CD16/32 (BioLegend) were preadded to block nonspecific binding of immunoglobulin to macrophage Fc receptors. After washes, the cells were subjected to analysis by flow cytometry Attune NxT (Life Technology).

\section{Quantitative real-time PCR}

For mRNA-level analysis, cells were lysed at the indicated time points, after which mRNA extraction was performed using Omega RNA Isolation Kit and cDNA synthesis using First Strand cDNA Synthesis Kit (Takara). Quantitative real-time PCR (RT-PCR) (StepOnePlus RT-PCR system; Applied biosystems, Life), was performed using SYBR green (Takara) and primer pairs as listed in table 1. The mRNA levels were normalized to housekeeping gene expression $(\Delta \mathrm{Ct}=\mathrm{Ct}$ target mRNA $-\mathrm{Ct}$ Gapdh mRNA), and folds were calculated compared with an control sample in $2^{-\Delta \Delta \mathrm{Ct}}$ method $(\Delta \Delta \mathrm{Ct}=\Delta \mathrm{Ct}$ sample $-\Delta \mathrm{CT}$ control mean).

\section{Tumor cell killing assay}

MDA-MB-231-TAMs or B16-TAMs were pretreated $\left(3 \sim 5 \times 10^{4}\right.$ cells/well) with $30 \mu \mathrm{g} / \mathrm{mL}$ LTF, M860, or LTF-IC for 24 hours, then cocultured with carboxyfluorescein diacetate succinimidyl ester (CFSE) labeled MDA-MB-231 or B16 cells $\left(2 \times 10^{4}\right)$ for 18 hours, and followed by analysis with Propidium Iodide (PI) staining by FACS. Results were expressed by the percentage of $\mathrm{CFSE}^{+} \mathrm{PI}^{+}$cells in total $\mathrm{CFSE}^{+}$cells.

\section{Analysis of tumor-infiltrating cells}

Tumor-bearing mice were sacrificed by cervical dislocation and their solid tumors were harvested. Tumors were minced in RPMI medium with $1 \mu \mathrm{g} / \mathrm{mL}$ collagenase (Sigma-Aldrich), 2 units/ml hyaluronidase (SigmaAldrich), and $0.1 \mu \mathrm{g} / \mathrm{mL}$ DNase (Sigma-Aldrich) for 1 hour, and incubated in the $37^{\circ} \mathrm{C} .{ }^{28}$ Then the cell suspension was centrifuged with Ficoll to get the mononuclear cells. For FACS analysis, collagenase-digested tumor cells were incubated with rat anti-mouse CD16/CD32 blocking antibodies $(4 \mu \mathrm{g} / \mathrm{mL})$ to block unspecific binding. After RBC lysis, tumor cells were centrifuged and washed with PBS, incubated with $10 \%$ FBS and anti-mouse

\begin{tabular}{|c|c|c|}
\hline Target mRNA & Forward primer(5'-3') & Reverse primer (5'-3') \\
\hline \multicolumn{3}{|l|}{ Human } \\
\hline GAPDH & GAAGGTGAAGGTCGGAGTC & GAAGATGGTGATGGGATTT \\
\hline TNFA & GGCTCCAGGCGGTGCTTG & CAGATAGATGGGCTCATACCA \\
\hline ARG-1 & GCAGAAGTCAAGAAGAACGG & GGTTGTCAGTGGAGTGTTG \\
\hline VEGF & TGCATT CACATTTGTTGTGC & AGACCCTGGTGGACATCTTC \\
\hline$C D 206$ & GAGCAAACATACCTGACAGGATTA & GGACTTCCTGGTAACCAGTTCA \\
\hline \multicolumn{3}{|l|}{ Mouse } \\
\hline GAPDH & GTGCTGAGTATGTCGTGGAGTCTAC & TTGCTGACAATCTTGAGTGAGTTG \\
\hline ARG-1 & GAACACGGCAGTGGCTTTAAC & TGCTTAGCTCTGTCTGCTTTGC \\
\hline VEGF & AGTCCCATGAAGTGATAAGTTCA & ATCCGCATGATCTGCATGG \\
\hline GZMB & TCCTGCTACTGCTGACCTTGTC & CTTGATCGAAAGTAAGGCCATGT \\
\hline iNOS & CAGCTGGGCTGTACAAACCTT & CATTGGAAGTGAAGCGGTTCG \\
\hline
\end{tabular}

ARG-1, arginase-1; TNFA, tumor necrosis factor alpha; VEGF, vascular endothelial growth factor. 
CD45-AF700, CD11b-PE-Cy7, F4/80-APC-Cy7, Gr-1-APC, CD206-FITC, and CD86-PE (Biolegend) for $30 \mathrm{~min}$. After washes, the cells were subjected to analysis by flow cytometry Attune NxT (Life Technology). For intracellular staining, tumor cells were fixed with fixation buffer (Biolegend) for $20 \mathrm{~min}$ at room temperature and permeabilized with Intracellular staining perm wash buffer (Biolegend) for $30 \mathrm{~min}$. Tumor-infiltrating TAMs (viable, $\mathrm{CD} 11 \mathrm{~b}^{+} \mathrm{CD} 11 \mathrm{c}^{-} \mathrm{F} 4 / 80^{+} \mathrm{Gr}-\mathrm{1}^{-}$) were assessed for $\mathrm{TNF} \alpha$ and IL10. For Foxp 3 staining, tumor cells were incubated with anti-CD3-FITC, anti-CD4-APC, and anti-Foxp 3-PE (Biolegend) for $30 \mathrm{~min}$ at room temperature and analyzed by flow cytometry Attune NxT (Life Technology).

\section{Tumor-infiltrating TAMs isolation}

Tumor tissues were collected and cut into small pieces in PBS. After centrifugation, enzyme digestion was performed with $1 \mu \mathrm{g} / \mathrm{mL}$ collagenase (Sigma-Aldrich), 2 units $/ \mathrm{mL}$ hyaluronidase (Sigma-Aldrich), and $0.1 \mu \mathrm{g} / \mathrm{mL}$ DNase (Sigma-Aldrich) for 1 hour. Then the cell suspension was centrifuged with Ficoll to get the mononuclear cells, CD11b ${ }^{+} \mathrm{CD} 11 \mathrm{c}-\mathrm{F} 4 / 80^{+} \mathrm{Gr}-1^{-}$cells were sorted to get tumor-infiltrating TAMs. For isolation of macrophages in ascites of breast cancer patients, ascites were centrifuged with Ficoll directly to get mononuclear cells, followed by staining and sorting for the $\mathrm{CD} 14^{+} \mathrm{CD} 206^{+}$cells.

\section{Statistical analysis}

All experiments were repeated at least three times and the results are expressed as mean \pm SD. Comparison of the data was performed using the Student's t-test. Log-rank (Mantel-Cox) test was used to analyze long-term survival curve. Significance was defined as a $\mathrm{p}<0.05 \%$.

\section{RESULTS}

\section{LTF-IC repolarized human TAMs to M1-like phenotype}

To overcome the shortage of patient-derived TAMs, peripheral blood monocytes cultured in cancer cellconditioned media are widely employed as TAMs in laboratory research. ${ }^{12} 17$ 38-40 Such TAMs share similar gene signature, phenotypic and functional characteristics with patient-derived TAMs. ${ }^{178-40}$ Therefore, we initiated our study with TAMs prepared using $\mathrm{CD} 14^{+}$peripheral blood monocytes after 7 day culture with a cytokine cocktail of recombinant human IL-4, IL-10 and M-CSF in the presence of $30 \%$ supernatants of human breast adenocarcinoma line cells MDA-MB-231. The resultant macrophages, MDA-MB-231-TAMs, adopted an M2 phenotype as evidenced by high expression of CD206, VEGF and ARG-1, low expression of CD86, as well as ability to make strong IL-10, but not TNF- $\alpha$, response to LPS stimulation (online supplementary figure S1). Interestingly, these macrophages responded to stimulation with LTF-IC, but not LTF or M860 alone, by producing large amount TNF- $\alpha$ and marginal IL-10, which is a hallmark of M1 response (figure 1A). More importantly, macrophages purified from ascites of breast cancer patients also exhibited a similar TNF- $\alpha /$ IL-10 secretion pattern in response to LTF-IC (figure 1B). On the contrary, LPS at $100 \mathrm{ng} / \mathrm{mL}$ induced strong IL-10 but little or no TNF- $\alpha$ responses by MDA-MB-231-TAMs and macrophages isolated from breast cancer patients (figure $1 \mathrm{~A}$ and $\mathrm{B}$ ). Unlike LTFIC, IC between chicken OVA and M562, an OVA-specific $\mathrm{mAb}$ previously produced by this group, ${ }^{36}$ was unable to induce TNF- $\alpha$ production by MDA-MB-231-TAMs or cancer patient-derived macrophages (figure 1A and B). These results indicate that Fc $\gamma \mathrm{R}$ triggering was necessary but not sufficient in effective activation of TAMs, and also that coligation of LTF receptor(s) (LTF-Rs) and Fc $\gamma \mathrm{R}(\mathrm{s})$ was of critical importance for the ability of LTF-IC in reversing TAMs to a M1-like phenotype.

LTF-IC treatment resulted in significant downregulation of M2 markers CD163 and CD206 and upregulation of M1 markers CD86 and HLA-DR in MDA-MB-231-TAMs (figure 1C), which is in line with our previous observation that LTF-IC skewed human M2 cell differentiating towards M1-like phenotype. ${ }^{36}$ It is noteworthy that variation of CD86 and HLA-DR expression levels in MDA231TAMs after LTF treatment was negligible (figure 1C), which is inconsistent with previous reports that LTF alone could increase CD86 and HLA-DR expression on monocytes and macrophages. ${ }^{41} 42$ One possible explanation for the discrepancy is the difference of LTF concentrations $(30 \mu \mathrm{g} / \mathrm{mL}$ here vs $100 \mu \mathrm{g} / \mathrm{mL}$ in previous reports) in different studies. Another possibility would be that TAMs are less sensitive than monocytes and dendritic cells to LTF stimulation (online supplementary figure S3). Earlier we also observed NO production by M860stimulated neutrophils. ${ }^{35}$ This, however, was not evident in MDA-MB-231-TAMs stimulated with either M860 alone or in the form of LTF-IC (figure 1D). Tumor-derived signals can activate macrophages for the expression of VEGF and ARG-1, which could support tumor growth by inducing neovascularization and providing substrates for cancer cell proliferation. ${ }^{17}$ Interestingly, level of VEGF and ARG-1 mRNA expression in LTF-IC-treated MDAMB-231-TAMs decreased significantly compared with that of control cells treated, or untreated, with LTF or M860 alone (figure 1E). We, thus, conclude that LTF-IC is able to induce polarization of TAMs to M1-like phenotype with proinflammatory and anticancer potential.

\section{Engagement of mCD14 and CD32a in LTF-IC-driven M2-M1 conversion}

Given our previous finding that the membrane-bound CD14 (mCD14)-TLR4 complex and CD32a-Syk axis play pivotal roles in LTF-IC-mediated M2 macrophage activation and conversion to M1 phenotype ${ }^{36}$ we hypothesized that LTF-IC might drive human TAM repolarization via similar mechanisms. Figure 2A shows that MDA-MB-231TAMs strongly expressed mCD14, low affinity activating Fc $\gamma$ receptors IIa (Fc $\gamma$ RIIa, ie, CD32a) and high affinity activating Fc $\gamma$ Rs I (CD64) and III (CD16). As shown in figure 2B, blocking mAbs against hCD14 or CD32a, but not CD16 or CD64, almost completely abrogated 
A

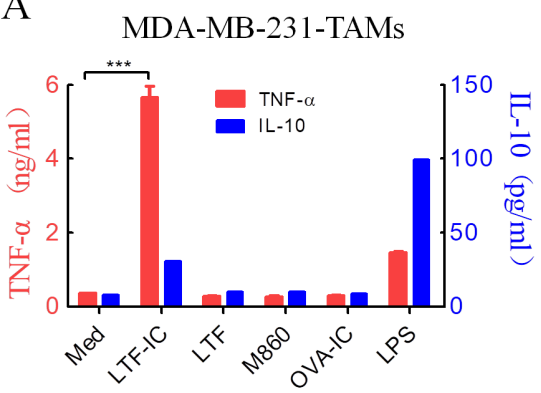

B

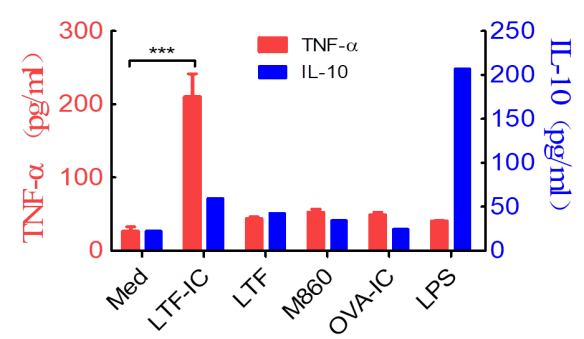

$\mathrm{C}$

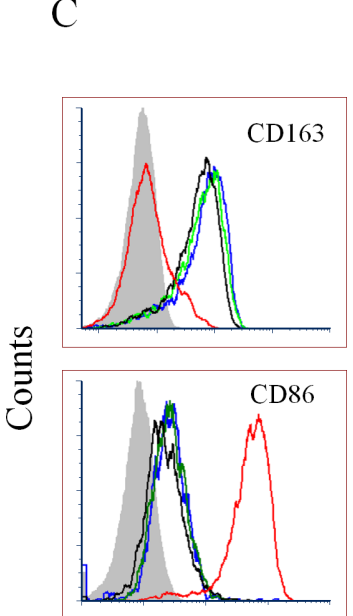

Fluorescence Intensity
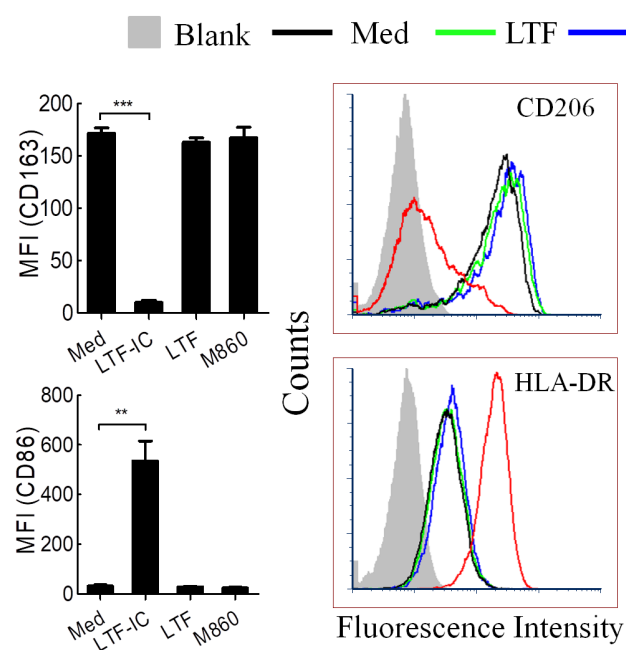

M860 - LTF-IC

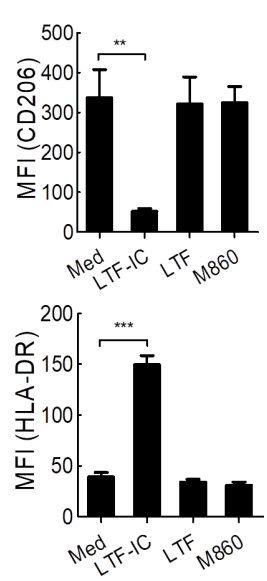

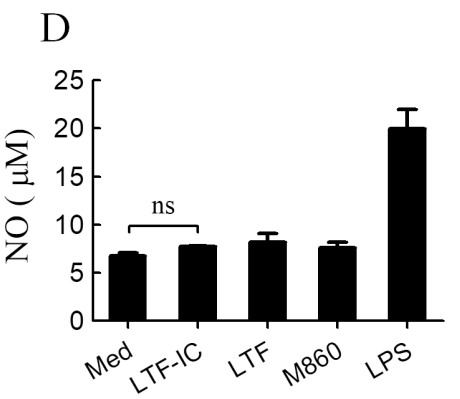

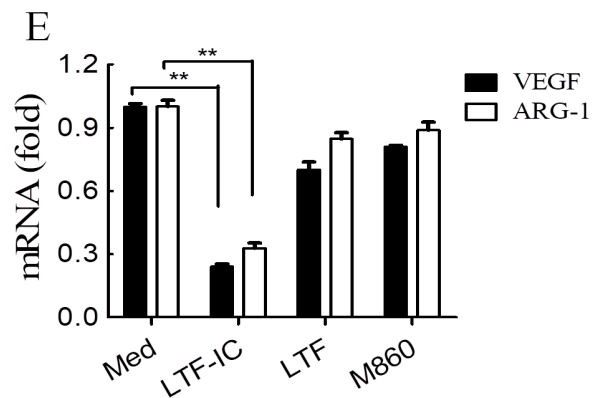

Figure 1 LTF-IC resets human TAMs to M1 phenotype. MDA-MB-231-TAMs derived from human peripheral blood monocytes (A) or macrophages purified from ascites of patients with breast cancer using CD14 microbeads (B) were treated in triplicate wells with LTF, M860, LTF-IC, OVA-IC $(30 \mu \mathrm{g} / \mathrm{mL})$ or LPS $(100 \mathrm{ng} / \mathrm{mL})$ for 24 hours and then the culture supernatants were collected for quantification of TNF- $\alpha$ and IL-10 using ELISA kits. Unstimulated cells were also included as negative control (Med). (C) Following 24 hours treatment with, or without (Med), LTF, M860 or LTF-IC (30 $\mu \mathrm{g} / \mathrm{mL})$, MDA-MB-231-TAMs were analyzed for surface expression of CD163, CD206, CD86 and HLA-DR using FACS. Representative histograms are presented, mean fluorescence intensity $(\mathrm{MFI} I \mathrm{SD})$ of cells from triplicate wells are shown in bar graphs on the right. (D) No concentration (mean \pm SD) in the culture supernatant of MDA-MB-231-TAMs after 24 hours stimulation with, or without (Med), LTF-IC, LTF, M860, or LPS was quantitated using the NO colorimetric assay kit. (E) VEGF and ARG-1 mRNA levels in MDA-MB-231-TAMs after 24 hours stimulation with, or without (Med), LTF-IC, LTF or M860 in triplicate wells were detected using Q-PCR. The results (mean $\pm \mathrm{SD}$ ), after normalization to GAPDH mRNA expression, are expressed as fold increase compared with the Med group. The results are representative of at least three experiments using cells from different donors. ${ }^{*} \mathrm{P}<0.05,{ }^{\star \star} \mathrm{P}<0.01,{ }^{\star \star \star} \mathrm{P}<0.001$. ARG-1, arginase-1; IC, immunocomplex; IL-10, interleukin-10; LPS, lipopolysaccharide; LTF, lactoferrin; OVA, ovalbumin; QPCR, quantitative PCR; TAMs, tumor-associated macrophages; TNF- $\alpha$, tumor necrosis factor $\alpha$; VEGF, vascular endothelial growth factor.

LTF-IC-induced TNF- $\alpha$ production by MDA-MB-231TAMs. Additionally, LTF-IC-induced downregulation of VEGF, ARG-1 and CD206 mRNA expression was effectively reversed by mAbs against either hCD14 or CD32a (figure 2C). These results confirm that LTF-IC drives human TAM conversion into M1-like phenotype via coligation of mCD14 and CD32a. 
A

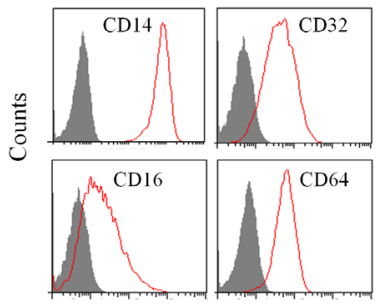

Fluorescence Intensity
B

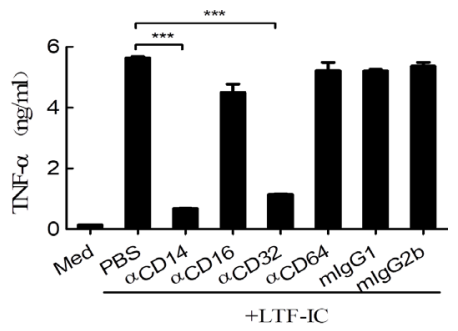

C

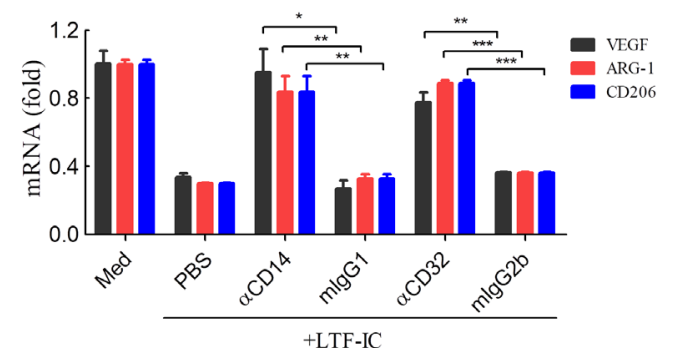

Figure 2 LTF-IC-driven TAM-M1 repolarization is dependent on mCD14 and CD32A. (A) MDA-MB-231-TAMs were stained with APC-labeled Abs against human CD14, CD16, CD32 or CD64 (red lines), or APC-labeled isotype control Abs (gray filled), for flow cytometric analysis. representative histograms are presented. (B, C) MDA-MB-231-TAMs were treated with LTF-IC $(30 \mu \mathrm{g} / \mathrm{mL})$ in the presence, or absence (PBS), of blocking Abs $(5 \mu \mathrm{g} / \mathrm{mL})$ against human CD14, CD16, CD32A or CD64 in triplicate wells. Irrelevant mouse IgG $\left(\mathrm{mlg}_{1}\right)$ and $\lg \mathrm{G} 2 \mathrm{~b}$ (mlgG2b) were included as specificity control, unstimulated cells (Med) were included as negative control. The culture supernatant was collected 24 hours later for quantitation of TNF- $\alpha$ using ELISA kit and the results expressed as mean concentration $(\mathrm{ng} / \mathrm{mL}) \pm S D(B)$. The cultured cells were harvested for analysis of VEGF, ARG-1 and CD206 mRNA expression using Q-PCR. The results, after normalized to GAPDH expression, are expressed as fold increase compared with the Med control). these are representatives of three experiments using blood samples from different donors. ${ }^{*} P<0.05,{ }^{\star *} P<0.01,{ }^{* \star} P<0.001$. ARG1, arginase-1; IC, immunocomplex; LTF, lactoferrin; PBS, phosphate-buffered saline; Q-PCR, quantitative PCR; TAMs, tumor-associated macrophages; TNF- $\alpha$, tumor necrosis factor $\alpha$; VEGF, vascular endothelial growth factor.

\section{LTF-IC-drives M1-like repolarization of hCD32a-TG mouse TAMs}

Unlike human monocytes/macrophages, murine macrophages were poor responders to LTF-IC stimulation in vitro, ${ }^{36}$ which could be explained, at least in part, by the absence of hCD32a homologue in mice. We, therefore, tested whether TAMs from hCD32a-TG mice (C57BL/6 background) would respond to LTF-IC stimulation by adopting an M1-like phenotype. Bone marrow cells were isolated from hCD32a-TG and C57BL/6 WT mice and differentiated into TAMs with cytokine cocktail of recombinant murine IL-4, IL-10 and M-CSF in the presence of $30 \%$ culture medium of B16 cell (a mouse melanoma line of $\mathrm{B} 6$ background). When the resultant hCD32a-TGB16-TAMs were treated with LTF-IC for 24 hours, level of CD206 and CD86 expression was strongly decreased

A
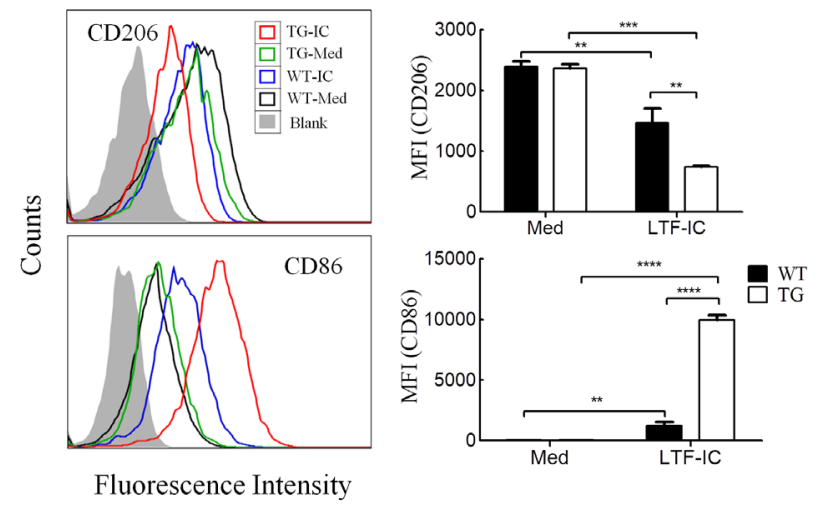

B
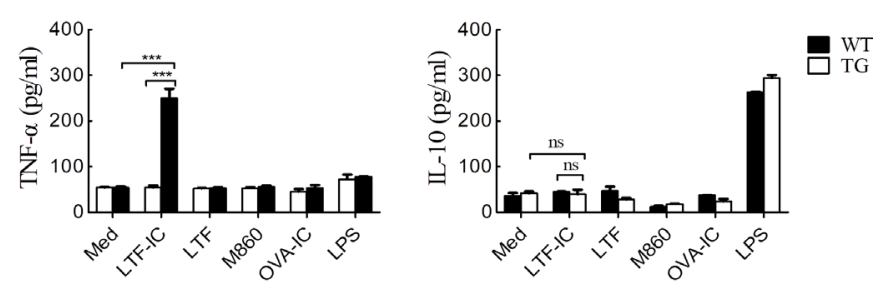

C
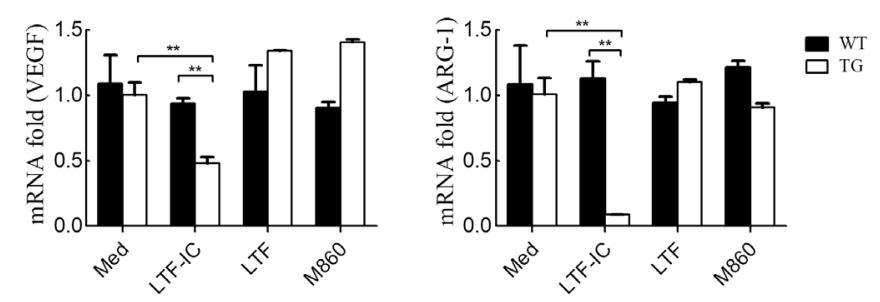

Figure 3 LTF-IC-driven repolarization of TAMs from hCD32a-TG mice. (A) TG-B16-TAMs and WT-B16-TAMs were treated with or without (Med), LTF-IC (TG-IC and WT-IC, respectively) in triplicate wells for 24 hours and then analyzed for CD206 and CD86 surface expression by FACS. Representative histograms are presented, and mean fluorescence intensity $\left(\mathrm{MFl}{ }_{ \pm} \mathrm{SD}\right)$ of cells from triplicate wells are summarized in bar graphs on the right. $(B, C)$ TG-B16-TAMs (TG) and WT-B16-TAMs (WT) were treated with, or without (Med), $30 \mu \mathrm{g} / \mathrm{mL}$ LTF-IC, LTF, M860, OVAIC or $100 \mathrm{ng} / \mathrm{mL}$ LPS for 24 hours followed by (B) ELISA quantitation of TNF $\alpha$ and IL-10 in the culture supernatants and (C) Q-PCR quantitation (less OVA-IC and LPS groups) of VEGF and ARG-1 the mRNA expression (normalized to GAPDH expression, expressed as fold increase compared with the Med control group). Data are representatives of three independent experiments. ${ }^{*} \mathrm{P}<0.05$, ${ }^{\star \star} \mathrm{P}<0.01$, ${ }^{\star \star *} \mathrm{P}<0.001$, ${ }^{* \star * *} \mathrm{P}<0.0001$. ARG-1, arginase-1; hCD32a, human CD32a; IC, immunocomplex; IL-10, interleukin-10; LPS, lipopolysaccharide; LTF, lactoferrin; OVA, ovalbumin; Q-PCR, quantitative PCR; TAMs, tumor-associated macrophages; TG, trangenic; TNF $\alpha$, tumor necrosis factor $\alpha$; VEGF, vascular endothelial growth factor; WT, wild type.

and upregulated, respectively (figure $3 \mathrm{~A}$ ), indicating a M1-like polarization. A similar trend in CD206 and CD86 expression variation was observed in LTF-IC-treated WT-B16-TAMs, but in a significantly smaller magnitude (figure 3A), suggesting a possibility that, in the absence of transgenic CD32a, co-ligation of mCD14 and mouse Fc $\gamma$ Rs by LTF-IC might also occur in TAMs from WT mice, 
thereby causing similar (but less strong) responses. Additionally, hCD32a-TG-B16-TAMs, but not WT-B16-TAMs, strongly responded to LTF-IC stimulation by producing TNF- $\alpha$ (figure 3B). In contrast to LPS, LTF-IC did not induce IL-10 production by either hCD32a-TG-B16-TAMs or WT-B16-TAMs (figure 3B). It is also of interest to note that B16-TAMs from hCD32a-TG, but not WT control, mice responded to LTF-IC stimulation by strongly downregulating VEGF and ARG-1 expression (figure 3C). Neither OVA-IC nor LTF or M860 alone could elicit TNF- $\alpha$ production, or VEGF and ARG-1 downregulation, in hCD32a-TG-B16-TAMs. These results do not only consolidate the importance of LTF-R-hCD32a co-ligation/crosssignaling in TAM repolarization but also provide solid basis for in vivo investigation on the therapeutic effect of LTF-IC using hCD32a-TG mouse model.

\section{LTF-IC rendered tumoricidal activity to TAMs via induction of GzmB}

M1 macrophages are known to kill tumor cells via secreted mediators (eg, TNFs and ROS) as well as phagocytosic attack requiring direct cell-cell contact (eg, antibodydependent cytotoxicity). When LTF-IC-pretreated human TAMs and hCD32a-TG mouse TAMs were cocultured with CFSE-labeled MDA-MB-231 or B16 cells, respectively, specific tumor cell lysis was observed by FACS analysis (figure 4A). An interesting finding here is that, following LTF-IC treatment for 24 hours, both human MDA-MB231-TAMs and hCD32a-TG-B16-TAMs secreted GzmB (figure 4B), which is a potent cytolytic enzyme ${ }^{43}$ and may directly contribute to the tumoricidal activity of LTF-IC-treated TAMs. This is collaborated by subsequent observation that Z-AAD-CMK, a GzmB-specific inhibitor, dose dependently inhibited specific lysis of tumor cells by LTF-IC-pretreated human and hCD32a-TG mouse TAMs (figure 4C).

\section{LTF-IC-mediated antitumor protection in hCD32a-TG mice}

Next, we asked if LTF-IC could exhibit antitumor effect by stimulating M1 polarization of TAMs in vivo. Viable B16 melanoma cells were implanted into hCD32a-TG or WT mice, followed by intraperitoneal administration of LTFIC, LTF, M860 or PBS. In B16 tumor-bearing hCD32a-TG mice, LTF-IC treatment significantly reduced tumor growth and prolonged animal survival compared with the groups treated, or untreated (PBS control), with LTF or M860 alone (figure 5A). LTF alone also resulted in slower tumor growth and prolonged survival than the PBS and M860 groups, but the difference was not statistically significant. In WT mice-bearing B16 solid tumors, LTF-ICtreatment also resulted in modest suppression (although not statistically significant) of tumor growth and increase of survival time than the PBS control group (figure 5A), echoing the in vitro results that WT-B16-TAMs responded to LTF-IC by modestly upregulating CD86 and downregulating CD206 (figure 3A). Thus LTF-IC might be able to induce M2-M1 shift in TAMs of WT mice by co-ligating mCD14 and mouse Fc $\gamma$ Rs, although the efficiency was not


Z-AAD-CMK $(\mu \mathrm{M})$

Figure 4 Tumoricidal activity of LTF-IC-treated TAMs correlates with GzmB production. (A) MDA-MB-231-TAMs (human) and hCD32a-TG-B16-TAMs (mouse) were pretreated with, or without (Med), LTF, M860, LTF-IC or OVA-IC in triplicate wells for 24 hours, and then mixed with CFSElabeled MDA-MB-231 or B16 tumor cells, respectively, and cocultured for another 18 hours (filled bars). CFSE-labeled MDA-MB-231 or B16 tumor cells cultured alone were included as controls (open bars). The cells were eventually harvested and subjected to PI staining for FACS analysis. Results are expressed as specific killing (percent CFSE ${ }^{+} \mathrm{PI}^{+}$ cells among the $\mathrm{CFSE}^{+}$population, mean $\pm \mathrm{SD}$ ) of target cells. (B) MDA-MB-231-TAMs (left panel), WT-B16-TAMs (WT) and TG-B16-TAMs (TG) (right panel) were treated with, or without (Med), LTF, M860, LTF-IC or OVA-IC in triplicate wells for 24 hours followed by ELISA quantitation of GzmB in the culture supernatant. (C) MDA-MB-231-TAMs (left panel) and hCD32a-TG-B16-TAMs (right panel) that had been pretreated with, or without (Med), LTF-IC for 24 hours were employed as killers against CFSE-labeled MDA-MB-231 or B16 cells, respectively, in the presence, or absence, of GzmB inhibitor Z-AAD-CMK. cells were acquired and analyzed by FACS after PI staining and the results expressed as specific killing (percent $\mathrm{CFSE}^{+} \mathrm{PI}^{+}$cells in the $\mathrm{CFSE}^{+}$population) of the tumor cells. Data are representatives of three experiments. ${ }^{\star} \mathrm{P}<0.05,{ }^{\star \star} \mathrm{P}<0.01,{ }^{\star \star *} \mathrm{P}<0.001$. hCD32a, human CD32a; IC, immunocomplex; LTF, lactoferrin; OVA, ovalbumin; PI, propidium iodide; TAM, tumor-associated macrophage; TG, trangenic; WT, wild type.

comparable to that in hCD32a-TG mice. To characterize TAMs in the TG and WT mice treated with LTF-IC or LTF and M860 alone, we sorted $\mathrm{CD} 11 \mathrm{~b}^{+} \mathrm{CD} 11 \mathrm{c} \mathrm{F} 4 / 80^{+} \mathrm{Gr}-\mathrm{l}^{-}$ macrophages from solid B16 tumor tissues and analyzed their expression of CD86, CD206, TNF- $\alpha$ and IL-10 by immunofluorescence staining. Quantitative analysis of the staining results indicates that, compared with that 
A
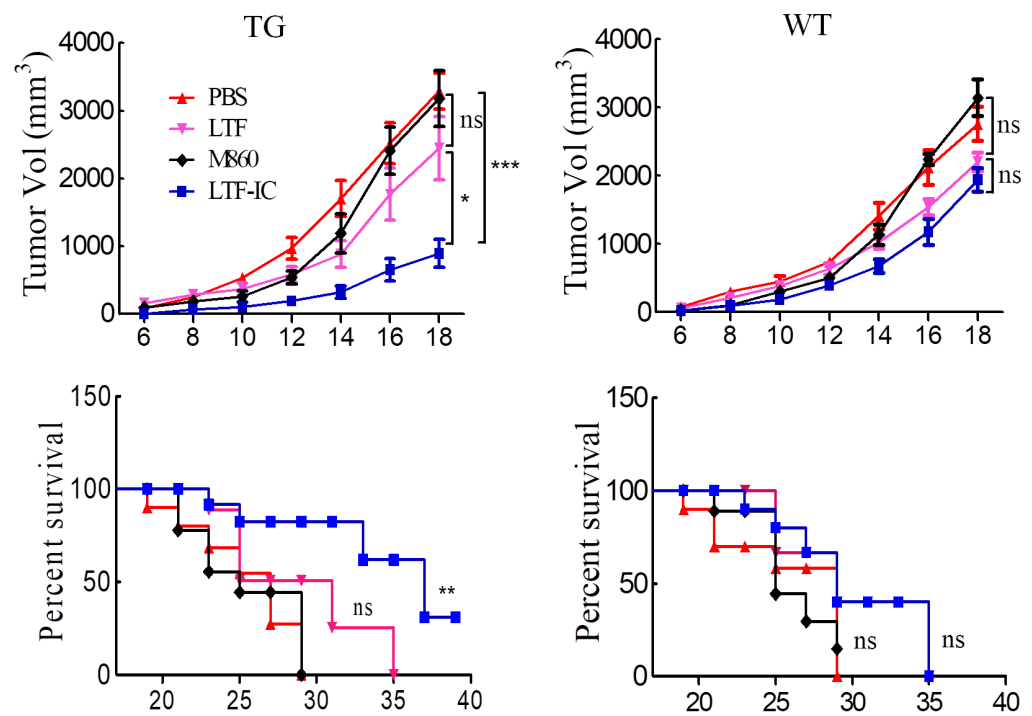

Days Post Implant

B
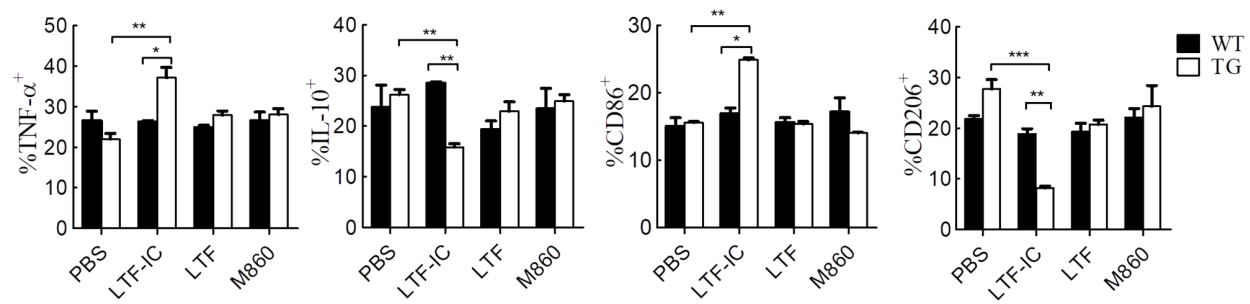

C
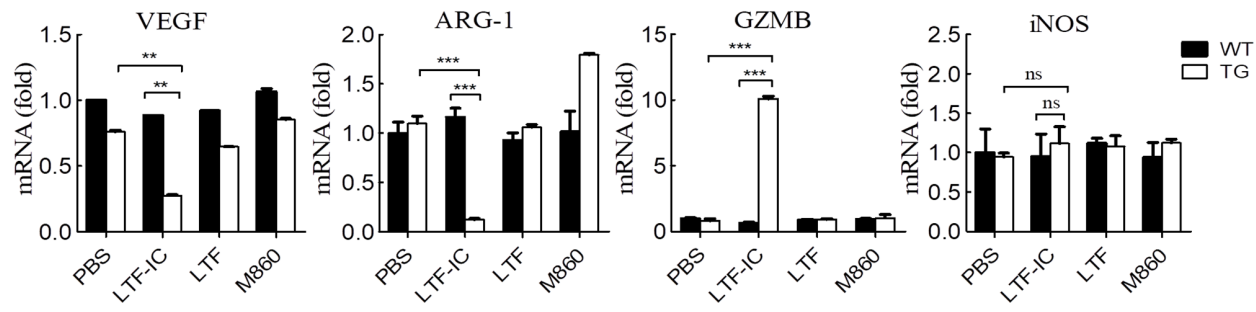

Figure 5 Antitumor effects of LTF-IC in vivo. (A) B16 tumor-bearing WT and hCD32a-TG mice ( $n=6$ ) were i.p. treated with or without (PBS), LTF, M860 or LTF-IC $\left(10 \mathrm{mg} \mathrm{kg}^{-1}\right)$ on days $-1,7$ and 14 . Volume of the solid tumors at the inoculation sites were measured every other day until day 18 (upper panels). Long-term survival of the tumor-bearing mice was recorded according to Kaplan-Meier analysis and analyzed by log-rank (Mantel-Cox) test (lower panels). (B) Additional groups of B16 tumor-bearing TG and WT mice $(n=3)$ similarly treated with PBS, LTF, M860 or LTF-IC were sacrificed, on day 18, for solid tumor tissues. Cells, thus, isolated were subjected to multicolor immunofluorescence staining and FACS analysis, gating on tumor-infiltrating CD11 $\mathrm{b}^{+} \mathrm{CD} 11 \mathrm{c}^{-} \mathrm{F} 4 / 80^{+} \mathrm{Gr}-1^{-}$TAMs expressing TNF- $\alpha$, IL-10, CD86 or CD206. Samples from each mouse were individually assessed, and the results are expressed as percent TNF- $\alpha-$, IL-10-, CD86- or CD206-positive cells among the CD11 $\mathrm{b}^{+} \mathrm{CD} 11 \mathrm{c}^{-} \mathrm{F} 4 / 80^{+} \mathrm{Gr}-1^{-}$population. (C) FACS sorted $\mathrm{CD} 11 \mathrm{~b}^{+} \mathrm{CD} 11 \mathrm{c}^{-} \mathrm{F} 4 / 80^{+} \mathrm{Gr}-1^{-} \mathrm{TAMs}$ from the above groups of TG and WT tumor-bearing mice were subjected to quantitative RT-PCR analysis for mRNA expression of VEGF, ARG-1, GZMB and iNOS. The results, normalized to GAPDH expression, are expressed as fold increase compared with TAMs from WT mice treated with PBS. ${ }^{*} \mathrm{P}<0.05,{ }^{* \star} \mathrm{P}<0.01,{ }^{* \star *} \mathrm{P}<0.001$. ARG-1, arginase-1; hCD32a, human CD32a; IC, immunocomplex; IL-10, interleukin-10; LTF, lactoferrin; PBS, phosphate-buffered saline; RT-PCR, real-time PCR; TAM, tumor-associated macrophage; TG, trangenic; TNF- $\alpha$, tumor necrosis factor $\alpha$; VEGF, vascular endothelial growth factor; WT, wild type.

from tumor-bearing mice given LTF or M860 alone, TAMs from LTF-IC-treated hCD32a-TG mice expressed significantly higher levels of TNF- $\alpha$ and CD86, and lower levels of CD206 and IL-10 (figure 5B). We also compared the sorted macrophages for mRNA expression of VEGF, ARG-1, GZMB and iNOS by Q-PCR. Decreased expression of VEGF and ARG-1, and strongly augmented expression of GZMB in cells from the LTF-IC-treated TG animals was observed (figure 5C). The enhanced GZMB expression supports the idea that GzmB could directly contribute to tumoricidal activity of LTF-IC-conditioned TAMs in vivo. On the other hand, no detectable change in iNOS 
expression level was observed, which is in line with the lack of NO production in LTF-IC-treated TAMs in vitro (figure 1D) and argues against ROS as an essential mediator in tumor cell killing by LTF-IC-treated TAMs in vivo.

\section{LTF-IC attenuated MDSC and Treg accumulation in solid tumor tissues}

Myeloid-derived suppressor cells (MDSCs) and $\mathrm{CD} 4^{+} \mathrm{Foxp}^{+}{ }^{+}$regulatory $\mathrm{T}$ cells (Tregs) are known as two major immunosuppressive cell types in tumor microenvironment. ${ }^{28}{ }^{44}$ It has been proposed that TAMs can interact with MDSCs and Tregs to form a 'vicious immunosuppressive triangle', which plays important roles in promoting tumor growth in patients. ${ }^{28}{ }^{44}$ We wondered whether such 'immunosuppressive triangle' could be interrupted by LTF-IC through repolarization of TAMs. As illustrated in figure $6 \mathrm{~A}, \mathrm{~B}$, the number of $\mathrm{CD} 11 \mathrm{~b}^{+} \mathrm{Gr}$ $1^{\text {hi }}$ MDSCs and CD $4^{+}{ }^{-}$oxp $3^{+}$Tregs within B16 tumor tissues from hCD32a-TG, but not WB control, mice markedly reduced after LTF-IC treatment. LTF or M860 alone did not show similar effects in either TG or WT mice. In addition, direct injection of LTF-IC-pretreated viable hCD32a-TG-B16-TAMs into solid tumors resulted in significant decrease of MDSCs and Tregs in the tumor tissues (figure 6C). Together these data suggest that the antitumor protective effect of LTF-IC is related to reduction of MDSCs and Trges in tumor microenvironment, likely mediated by repolarized TAMs.

\section{DISCUSSION}

TAMs are major infiltrating leukocytes in tumor microenvironment that generally display an M2-like phenotype with immunosuppressive activity and may contribute to the formation and progression of cancer. ${ }^{7811}$ Therefore, repolarization of TAMs to an anti-tumor M1-like phenotype represents an attractive strategy for tumor immunotherapies. ${ }^{21-27}$ In this study, we have demonstrated for the first time that, through costimulation of mCD14 and hCD32a, LTF-IC is able to drive the conversion of TAMs into M1-like phenotype characterized by strong production of TNF- $\alpha$ and GzmB and potent tumoricidal activity. The hCD32a transgene enabled mouse TAMs responding to LTF-IC in a fashion similar to human macrophages. Administration of LTF-IC significantly suppressed tumor growth and prolonged animal survival of B16 tumorbearing hCD32a-TG mice, which is underlined by the accumulation of M1-like macrophages and reduction of immunosuppressive MDSCs and Tregs in the tumor tissues. These data strongly support our hypothesis that LTF-IC-induced M2-to-M1 switch could be therapeutically useful in the treatment of cancers.

Direct antitumor effects of LTF and its derivatives have previously been reported, including inhibition of cancer cell proliferation, cell cycle arrest and facilitation of cancer cell apoptosis or necrosis. ${ }^{30-34}$ In our study, however, neither LTF alone nor LTF-IC showed direct antitumor effects in terms of proliferation inhibition (online supplementary figure S2) or specific lysis (figure 4A) of tumor cells. One possible explanation for this discrepancy is the huge variation of LTF concentrations employed by different groups. The LTF or LTF-IC concentration used in the present work was $30 \mu \mathrm{g} / \mathrm{mL}$, which is close to the pathophysiological range of LTF in vivo but much lower than that (often more than $300 \mu \mathrm{g} / \mathrm{mL}$ ) in previous studies by other groups. ${ }^{34}$

It has also been shown that LTF exerted antitumor effects through regulating the immune system, such as induction of IL-18 production, upregulation of NK activity, or increase the expression of CD86 and HLA-DR on monocytes and dendritic cells. ${ }^{41}{ }^{42}$ In our hands, however, LTF alone was by far not comparable to LTF-IC in activating human monocytes, monocyte-derived macrophages, as documented in our earlier work, ${ }^{45}$ or TAMs as shown here (figure 1C, online supplementary figure S3). Increased GZMB gene expression in tumor infiltrating macrophages from LTF-IC-treated TG mice is suggestive of GzmB involvement in LTF-IC-mediated antitumor protective effects in vivo. Earlier we reported that M860 could induce ROS production by human neutrophils in vitro. ${ }^{35}$ In the present study, however, TAMs showed neither enhanced iNOS gene expression nor NO production after LTF-IC-treatment in vitro and in vivo.

It is evident that the potent activity of LTF-IC in TAM repolarization derives from its ability in triggering intracellular cross-signaling between $\mathrm{hFc} \gamma \mathrm{RIIa}$ and mCD14/ TLR4. There are earlier reports showing that ordinary (ie, non-biologically antigen-containing) IgG ICs could promote macrophage-mediated tumor cell killing in a Fc $\gamma$ R-dependent manner. ${ }^{46-48}$ Recently, Domínguez-Soto et al documented that intravenous IgG, a preparation of polyclonal and polyspecific Igs derived from the plasma of thousands of healthy donors, caused a M2-to-M1 polarization. ${ }^{26}$ These results contradict our suggestion that biologically active antigen-containing IgG ICs (such as LTF-IC) rather than ordinary ICs possess the ability to drive the M2-M1 conversion of TAMs. Again it should be noted that very high concentrations of ICs or IgG $\left(10 \mathrm{mg} / \mathrm{mL}\right.$ in vitro and $100 \mathrm{mg} \mathrm{kg}^{-1}$ in vivo) were used in above-mentioned studies. ${ }^{26}{ }^{46-48}$ Our evidence argues that biologically active antigen-containing ICs such as LTF-IC exhibit extraordinarily strong activity on TAMs by triggering cross-signaling between hFc $\gamma$ RIIa and LTF-R (eg, mCD14/TLR4). A number of biologically active autoantigen-containing IgG ICs capable of triggering crosstalk between TLRs and Fc $\gamma$ Rs have been reported. ${ }^{49}$ Some of them could serve as additional candidates with ability to repolarize TAMs towards M1 phenotype in vivo.

A puzzling earlier observation of this group was that LTF-IC only strongly activated human but not mouse monocytes/macrophages ${ }^{36}$ It is now clear that this was due to the lack of hCD32a (Fc $\gamma$ RIIa) homologue in mouse. Mice express four different classes of Fc $\gamma$ Rs known as Fc $\gamma R I$, Fc $\gamma R I I B, F c \gamma R I I I$ and Fc $\gamma R I V$, while human Fc $\gamma R$ system is more complex including Fc $\gamma$ RI, Fc $\gamma$ RIIA-C and Fc $\gamma$ RIIIA-B ${ }^{50}$ Mouse Fc $\gamma$ RIII is close to human Fc $\gamma$ RIIa, 
A

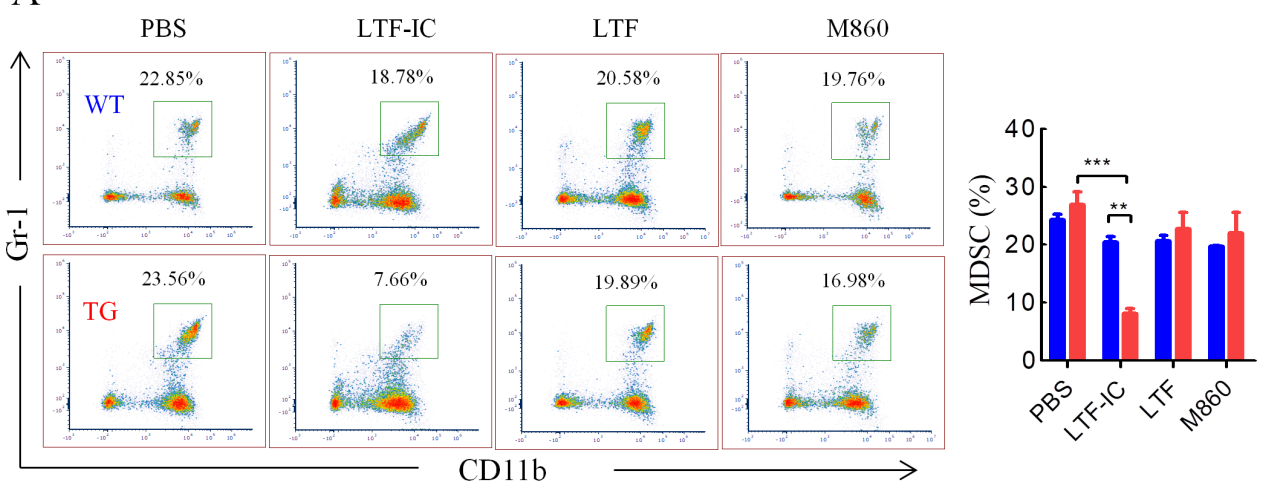

$\mathrm{B}$

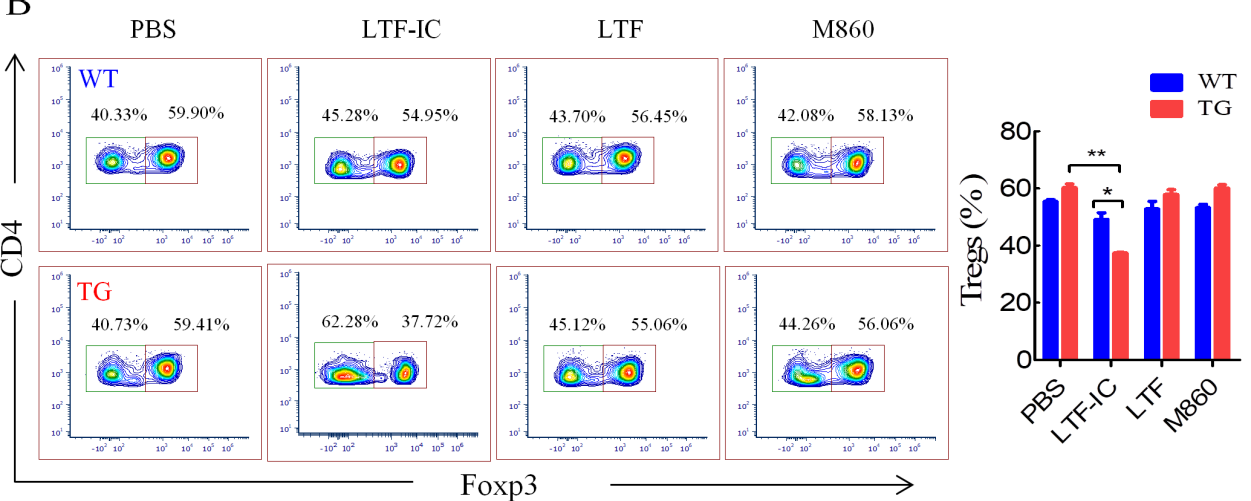

$\mathrm{C}$
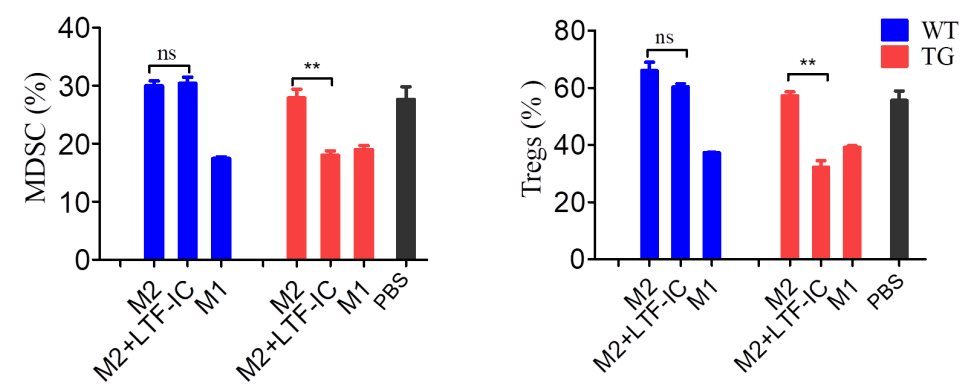

Figure 6 Negative correlation between tumor-infiltrating M2-like TAMs, MDSCs and Tregs after LTF-IC treatment in TG mice. B16 melanoma-bearing WT and hCD32a-TG mice $(n=3)$ were i.p. treated with or without (PBS), LTF, M860 or LTF-IC (10 mg $\mathrm{kg}^{-1}$ ) on days $-1,7$ and 14 . The mice were sacrificed, on day 18 , for solid tumor tissues. cells thus isolated were subjected to multicolor immunofluorescence staining and FACS analysis, gating on populations of (A) CD $45^{+} \mathrm{F} 4 / 80^{-} \mathrm{CD} 11 \mathrm{~b}^{+} \mathrm{Gr}-1^{+}$(MDSC) and (B) $\mathrm{CD}^{+} \mathrm{Foxp}^{+}$(Treg) cells. Melanoma samples from each mouse were individually assessed. in addition to representative dot plots presented, the results (mean+SD) are also shown as percent MDSCs and Trges among the CD $45^{+}$and $C D 4^{+}$population, respectively, in the bar graphs on the right. (C) C57BL/6 mice $(n=3)$ bearing B16 melanoma were intratumorally injected with either PBS, or LTF-IC-treated WT-/TG-B16-TAMs (M2 +LTFIC) or WT/TG mouse bone marrow-derived M1 macrophages (M1) every 2 days $\left(1 \times 10^{5}\right.$ cells per inoculation). Mice were sacrificed on day 18 and tumor tissue-extracted cells were subjected to multicolor immunofluorescence staining and FACS analysis, gating on $\mathrm{CD} 45^{+} \mathrm{F} 4 / 80^{-} \mathrm{CD} 11 \mathrm{~b}^{+} \mathrm{Gr}-1^{+}$(MDSC) and $\mathrm{CD} 4^{+} \mathrm{Foxp} 3^{+}$ (Treg) cells. The results (mean+SD) are expressed as percent MDSCs and Tregs among the CD45 $5^{+}$or $C D 45^{+} \mathrm{CD} 3^{+} \mathrm{CD} 4^{+}$ populations, respectively. ${ }^{\star} \mathrm{P}<0.05,{ }^{* *} \mathrm{P}<0.01,{ }^{* \star *} \mathrm{P}<0.001$. hCD32a, human CD32a; IC, immunocomplex; LTF, lactoferrin; PBS, phosphate-buffered saline; TAM, tumor-associated macrophage; TG, trangenic; WT, wild type.

but it lacks the immunoreceptor tyrosine-based activation motif-containing intracellular domain present in hFc $\gamma$ RIIa. ${ }^{50}$

Fine-tuning the immune status in tumor microenvironment for the purpose of antitumor therapy requires effective downregulation of immunosuppressive TAMs, MDSCs, Tregs and upregulation of immune-active $\mathrm{CD}^{+}$
T cells and NK cells. ${ }^{44}{ }^{51-53}$ We have demonstrated that LTF-IC treatment not only converted TAMs to proinflammatory M1-like macrophages with tumoricidal activity but also decreased MDSC and Treg cell abundance in tumor microenvironment. Although there is no evidence showing that LTF-IC could directly target $\mathrm{T}$ cells, our previous study found that LTF-IC-pretreated M2 
macrophages induced $\mathrm{T}$ cell polarization towards $\mathrm{Th} 1$ subset and produced large amount IFN $-\gamma .{ }^{36}$ Whether this mechanism was leveraged by LTF-IC in fighting against tumor remains to be further investigated.

\section{CONCLUSIONS}

Through coligation of mCD14/TLR4 and Fc $\gamma$ RIIa, LTF-IC effectively drives TAMs repolarization toward M1-like phenotype with tumoricidal activity. The in vivo antitumor protective effects of LTF-IC are attributable to augmentation of M1-like macrophages and inhibition of immunosuppressive MDSC and Tregs in tumor tissues. LTF-IC-induced M2-to-M1 switch might be therapeutically useful in the treatment of cancers.

Present affiliations The present affiliation of Chenhui Gao is: Department of Nuclear Medicine, Second Affiliated Hospital of Soochow University, Suzhou, China.

Contributors $H D$ and $X G$ designed the research. $H D, Y Y, C G$ and HS carried out the experiment. $H D$ analyzed the data. $H D$ and $X G$ prepared the manuscript. All authors discussed the results and commented on the manuscript.

Funding This work was supported by grants from National Key Research and Development Program of China (No. 2017YFA0104502) and the Natural Science Foundation of China (31770942/31570868).

Competing interests None declared.

Patient consent for publication Not required.

Ethics approval All protocols were approved by the Medical Ethical Committee of Soochow University.

Provenance and peer review Not commissioned; externally peer reviewed.

Data availability statement Data are available in a public, open access repository. Data are available on reasonable request. All data relevant to the study are included in the article or uploaded as supplementary information. The data used and/or analyzed for this study is available from the corresponding author at reasonable request.

Open access This is an open access article distributed in accordance with the Creative Commons Attribution Non Commercial (CC BY-NC 4.0) license, which permits others to distribute, remix, adapt, build upon this work non-commercially, and license their derivative works on different terms, provided the original work is properly cited, appropriate credit is given, any changes made indicated, and the use is non-commercial. See http://creativecommons.org/licenses/by-nc/4.0/.

\section{ORCID iDs}

Hongliang Dong http://orcid.org/0000-0002-6820-9105

Chenhui Gao http://orcid.org/0000-0002-5775-8539

Chao Hong http://orcid.org/0000-0002-5501-1108

\section{REFERENCES}

1 Murray PJ, Allen JE, Biswas SK, et al. Macrophage activation and polarization: Nomenclature and experimental guidelines. Immunity 2014;41:14-20.

2 Sica A, Mantovani A. Macrophage plasticity and polarization: in vivo veritas. J Clin Invest 2012;122:787-95.

3 Shapouri-Moghaddam A, Mohammadian S, Vazini H, et al. Macrophage plasticity, polarization, and function in health and disease. J Cell Physiol 2018;233:6425-40.

4 Martinez FO, Sica A, Mantovani A, et al. Macrophage activation and polarization. Front Biosci 2008;13:453-61.

5 Shrivastava R, Shukla N. Attributes of alternatively activated (M2) macrophages. Life Sci 2019;224:222-31.

6 Pathria P, Louis TL, Varner JA. Targeting tumor-associated macrophages in cancer. Trends Immunol 2019;40:310-27.

7 Chanmee T, Ontong P, Konno K, et al. Tumor-Associated macrophages as major players in the tumor microenvironment. Cancers 2014;6:1670-90.
8 Noy R, Pollard JW. Tumor-Associated macrophages: from mechanisms to therapy. Immunity 2014;41:49-61.

9 Qian B-Z, Li J, Zhang H, et al. Ccl2 recruits inflammatory monocytes to facilitate breast-tumour metastasis. Nature 2011;475:222-5.

10 Franklin RA, Liao W, Sarkar A, et al. The cellular and molecular origin of tumor-associated macrophages. Science 2014;344:921-5.

11 Solinas G, Germano G, Mantovani A, et al. Tumor-associated macrophages (TAM) as major players of the cancer-related inflammation. J Leukoc Biol 2009;86:1065-73.

12 Sousa S, Brion R, Lintunen M, et al. Human breast cancer cells educate macrophages toward the M2 activation status. Breast Cancer Res 2015;17:101.

13 Greaves P, Gribben JG. The role of B7 family molecules in hematologic malignancy. Blood 2013;121:734-44.

14 Tirapu I, Huarte E, Guiducci C, et al. Low surface expression of B7-1 (CD80) is an immunoescape mechanism of colon carcinoma. Cancer Res 2006;66:2442-50.

15 Kren L, Muckova K, Lzicarova E, et al. Production of immunemodulatory nonclassical molecules HLA-G and HLA-E by tumor infiltrating ameboid microglia/macrophages in glioblastomas: a role in innate immunity? J Neuroimmunol 2010;220:131-5.

16 Adeegbe DO, Nishikawa $\mathrm{H}$. Natural and induced T regulatory cells in cancer. Front Immunol 2013;4:190.

17 Colegio OR, Chu N-Q, Szabo AL, et al. Functional polarization of tumour-associated macrophages by tumour-derived lactic acid. Nature 2014;513:559-63.

18 Arlauckas SP, Garren SB, Garris CS, et al. Arg1 expression defines immunosuppressive subsets of tumor-associated macrophages. Theranostics 2018;8:5842-54.

19 Yang J, Yan J, Liu B. Targeting VEGF/VEGFR to modulate antitumor immunity. Front Immunol 2018;9:978.

20 Yang L, Zhang Y. Tumor-Associated macrophages: from basic research to clinical application. J Hematol Oncol 2017;10:58.

21 Lee C, Jeong H, Bae Y, et al. Targeting of M2-like tumor-associated macrophages with a melittin-based pro-apoptotic peptide. $J$ Immunother Cancer 2019;7:147.

22 Di Mitri D, Mirenda M, Vasilevska J, et al. Re-Education of tumorassociated macrophages by CXCR2 blockade drives senescence and tumor inhibition in advanced prostate cancer. Cell Rep 2019;28:2156-68.

23 Pyonteck SM, Akkari L, Schuhmacher AJ, et al. Csf-1R inhibition alters macrophage polarization and blocks glioma progression. Nat Med 2013;19:1264-72.

24 Ruffell B, Coussens LM. Macrophages and therapeutic resistance in cancer. Cancer Cell 2015;27:462-72.

25 Andón FT, Digifico E, Maeda A, et al. Targeting tumor associated macrophages: the new challenge for nanomedicine. Semin Immunol 2017;34:103-13.

26 Domínguez-Soto A, de las Casas-Engel M, Bragado R, et al. Intravenous immunoglobulin promotes antitumor responses by modulating macrophage polarization. J Immunol 2014;193:5181-9.

27 Liu M, Luo F, Ding C, et al. Dectin-1 activation by a natural product $\beta$-glucan converts immunosuppressive macrophages into an M1-like phenotype. J Immunol 2015; 195:5055-65.

28 Wiehagen KR, Girgis NM, Yamada DH, et al. Combination of CD40 agonism and CSF-1R blockade Reconditions tumor-associated macrophages and drives potent antitumor immunity. Cancer Immunol Res 2017:5:1109-21.

29 Chen D, Xie J, Fiskesund R, et al. Chloroquine modulates antitumor immune response by resetting tumor-associated macrophages toward M1 phenotype. Nat Commun 2018;9:873.

30 González-Chávez SA, Arévalo-Gallegos S, Rascón-Cruz Q. Lactoferrin: structure, function and applications. Int J Antimicrob Agents 2009;33:301.e1-301.e8.

31 Wei L, Zhang X, Wang J, et al. Lactoferrin deficiency induces a pro-metastatic tumor microenvironment through recruiting myeloidderived suppressor cells in mice. Oncogene 2020;39:122-35.

$32 \mathrm{Hu} \mathrm{L}$, Gao C-H, Hong C, et al. Expression, purification, and breast cancer cell inhibiting effect of recombinant human lactoferrin C-lobe. Biosci Biotechnol Biochem 2016;80:257-63.

33 Siqueiros-Cendón T, Arévalo-Gallegos S, Iglesias-Figueroa BF, et al. Immunomodulatory effects of lactoferrin. Acta Pharmacol Sin 2014;35:557-66.

34 Zhang Y, Lima CF, Rodrigues LR. Anticancer effects of lactoferrin: underlying mechanisms and future trends in cancer therapy. Nutr Rev 2014;72:763-73.

35 Hu X-M, Xu Y-R, Yan R, et al. A novel murine anti-lactoferrin monoclonal antibody activates human polymorphonuclear leukocytes through membrane-bound lactoferrin and TLR4. Biomed Res Int 2015;2015:285237. 
36 Gao C-H, Dong H-L, Tai L, et al. Lactoferrin-Containing immunocomplexes drive the conversion of human macrophages from M2- into M1-like phenotype. Front Immunol 2018;9:37.

37 Benner B, Scarberry L, Suarez-Kelly LP, et al. Generation of monocyte-derived tumor-associated macrophages using tumorconditioned media provides a novel method to study tumorassociated macrophages in vitro. J Immunother Cancer 2019;7:140.

38 Solinas G, Schiarea S, Liguori M, et al. Tumor-conditioned macrophages secrete migration-stimulating factor: a new marker for M2-polarization, influencing tumor cell motility. J Immunol 2010;185:642-52.

39 Wenes M, Shang M, Di Matteo M, et al. Macrophage metabolism controls tumor blood vessel morphogenesis and metastasis. Cell Metab 2016;24:701-15.

40 Grugan KD, McCabe FL, Kinder M, et al. Tumor-associated macrophages promote invasion while retaining Fc-dependent antitumor function. J Immunol 2012;189:5457-66.

41 Hwang S-A, Actor JK. Lactoferrin modulation of BCG-infected dendritic cell functions. Int Immunol 2009;21:1185-97.

42 Hwang S-A, Kruzel ML, Actor JK. Influence of bovine lactoferrin on expression of presentation molecules on BCG-infected bone marrow derived macrophages. Biochimie 2009;91:76-85.

43 Rousalova I, Krepela E. Granzyme B-induced apoptosis in cancer cells and its regulation (review). Int $J$ Oncol 2010;37:1361-78.

44 Munn DH, Bronte V. Immune suppressive mechanisms in the tumor microenvironment. Curr Opin Immunol 2016;39:1-6.
$45 \mathrm{Hu} \mathrm{L}$, Hu X, Long K, et al. Extraordinarily potent proinflammatory properties of lactoferrin-containing immunocomplexes against human monocytes and macrophages. Sci Rep 2017;7:4230.

46 Hogarth PM, Pietersz GA. Fc receptor-targeted therapies for the treatment of inflammation, cancer and beyond. Nat Rev Drug Discov 2012;11:311-31.

47 Guilliams M, Bruhns P, Saeys Y, et al. The function of Fcy receptors in dendritic cells and macrophages. Nat Rev Immunol 2014;14:94-108.

48 Ravetch J. In vivo veritas: the surprising roles of Fc receptors in immunity. Nat Immunol 2010;11:183-5.

49 Marshak-Rothstein A, Rifkin IR. Immunologically active autoantigens: the role of Toll-like receptors in the development of chronic inflammatory disease. Annu Rev Immunol 2007;25:419-41.

50 Nimmerjahn F, Ravetch JV. Fcgamma receptors as regulators of immune responses. Nat Rev Immunol 2008;8:34-47.

51 Chauhan VP, Chen IX, Tong R, et al. Reprogramming the microenvironment with tumor-selective angiotensin blockers enhances cancer immunotherapy. Proc Natl Acad Sci U S A 2019;116:10674-80.

52 Travers M, Brown SM, Dunworth M, et al. Dfmo and 5-azacytidine increase M1 macrophages in the tumor microenvironment of murine ovarian cancer. Cancer Res 2019;79:3445-54.

53 da Silva JL, Dos Santos ALS, Nunes NCC, et al. Cancer immunotherapy: the art of targeting the tumor immune microenvironment. Cancer Chemother Pharmacol 2019;84:227-40. 